\title{
Le bois dit de Danemarche dans le décor de la demeure aristocratique vers 1400
}

\section{Alain Salamagne}

\section{Q OpenEdition}

1 Journals

Édition électronique

URL : https://journals.openedition.org/edl/3362

DOI : $10.4000 /$ edl.3362

ISSN : 2296-5084

Éditeur

Université de Lausanne

\section{Édition imprimée}

Date de publication : 30 novembre 2020

Pagination : 135-154

ISBN : 978-2-940331-75-8

ISSN : 0014-2026

\section{Référence électronique}

Alain Salamagne, "Le bois dit de Danemarche dans le décor de la demeure aristocratique vers 1400 », Études de lettres [En ligne], 314 | 2020, mis en ligne le 15 décembre 2021, consulté le 16 décembre 2021. URL : http://journals.openedition.org/edl/3362 ; DOI : https://doi.org/10.4000/edl.3362 


\section{LE BOIS DIT DE DANEMARCHE DANS LE DÉCOR DE LA DEMEURE ARISTOCRATIQUE VERS 1400}

Le rôle du lambrissage dans le décor intérieur de la demeure aristocratique reste encore peu connu. Pourtant les comptabilités, et quelques ensembles conservés comme celui du château royal de Vincennes, montrent l'importance qui était accordée à ces décors comme une marque du statut aristocratique de la demeure noble. Certains lambris dits de Illande, Ollande ou Danemarche, termes qui renvoient à leur origine géographique, la Livonie, ou à leur transit commercial par la Hollande ou le Danemark, étaient particulièrement recherchés. Si ce type de décor fut mis en œuvre dès le début $\mathrm{du} \mathrm{XIV}^{\mathrm{e}}$ siècle dans la demeure aristocratique, le relais des chantiers royaux après 1350 - probablement depuis les régions les plus septentrionales -, semble avoir été essentiel dans sa réception puis sa diffusion dans les logis princiers, particulièrement en Bourgogne, Berry et Poitou.

Les travaux de restauration de la tour maîtresse du château royal de Vincennes ont attiré l'attention ces dernières années sur les lambris qui recouvraient les murs ou les voûtes de certaines pièces, $150 \mathrm{~m}^{2}$ ayant été conservés sur les voûtes de la chambre de la reine au premier étage et au deuxième étage dans la chambre du roi, la tourelle sud-ouest, qui servait de garde-robe (fig. 1), l'oratoire, les latrines et l'étude en encorbellement. Ces lambris, provenant de chênes des forêts de la Baltique qui avaient été abattus entre 1367 et 1371, furent posés après $1370^{1}$. Qualifiés dans les comptes des $\mathrm{XIV}^{\mathrm{e}}$ et $\mathrm{XV}^{\mathrm{e}}$ siècles d'"aiisselles", "aiis" (planches de chêne) ou "bors» (du néerlandais "bord", "bort» qu'on retrouve dans l'anglais «board») «d'Illande», «d'Ollande», de «Danemarche», «d'Alemarche»,

I. J. Chapelot, D. Pousset, «Les lambris du donjon». 


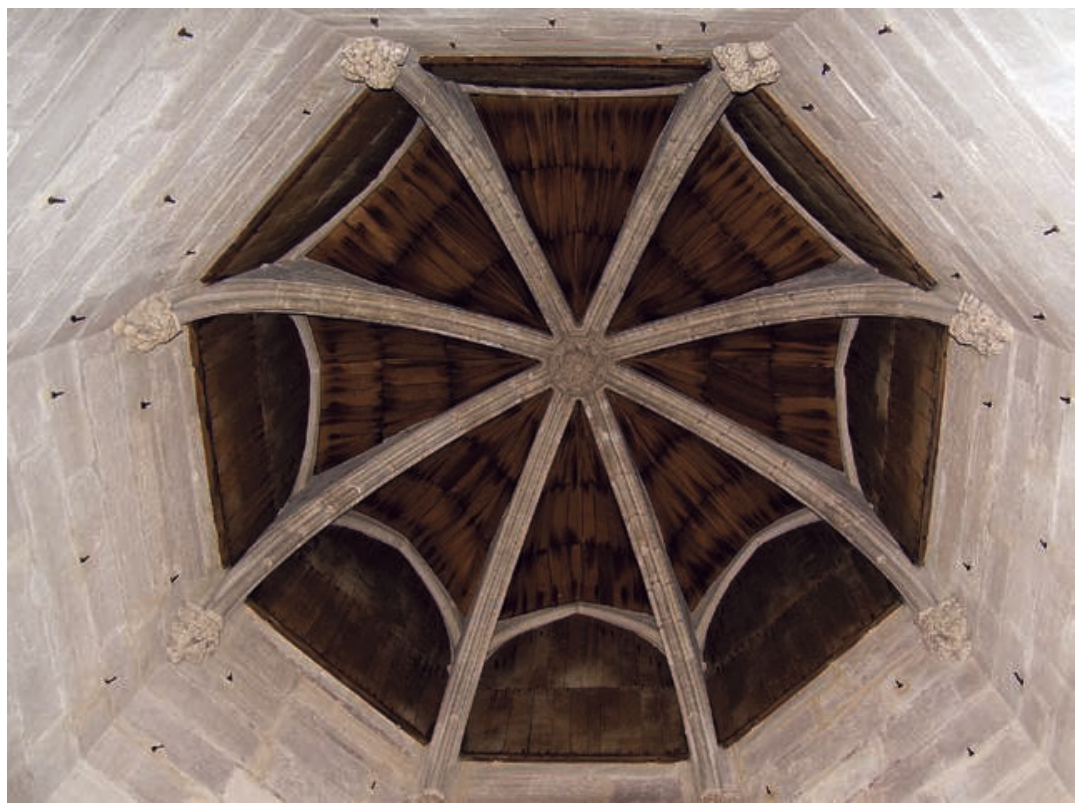

Fig. 1 - Vincennes, tour maîtresse, second étage, tourelle sud-ouest : voûte lambrissée. Photographie A. Salamagne.

«Dalemagne», on les a donc dits provenir du Danemark, d'Irlande, de Hollande.

Le premier qualificatif "Illande» renvoie en fait à la Livonie, appelée Livland à l'époque médiévale comme dans l'allemand actuel, région qui au XII ${ }^{\mathrm{e}}$ siècle désignait un territoire compris entre l'Estonie au nord et la Lettonie au sud (avec le port de Riga). Les autres qualificatifs "d'Ollande» et "Danemarche» 2 s'expliquent probablement par le relais de la Hollande ou du Danemark sur les routes maritimes reliant Bruges à la Baltique. On notera encore le rôle possible joué par la dynastie des comtes de Hainaut-Hollande dans ce transfert. Enfin, la mention "Dalemagne» doit renvoyer aux acteurs de ce commerce, les marchands des ports des villes de la Hanse, de Brême, Dantzig, Königsberg, Lübeck, Hambourg par lesquels se faisaient ces importations vers Bruges et son port de l'Écluse («Sluis»), avérées dès les années 1360-1380³. Les villes

2. Ainsi orthographié dans les chroniques du temps.

3. Ph. Lardin, Les chantiers du bâtiment en Normandie orientale à la fin $d u$ Moyen Âge, p. 41. 
d'Anvers $^{4}$ et de Gand ${ }^{5}$ pouvaient constituer des centres de redistribution secondaires. Jean-Pierre Sosson a noté pour les XIV et $\mathrm{XV}^{\mathrm{e}}$ siècles à Bruges le fort courant d'importation des bois depuis la Hollande, l'Allemagne, la Prusse, le bassin de la Baltique, la Scandinavie enfin ${ }^{6}$.

Ainsi, en 1423, Jehan Bouchout, "marchant natif du pays d'Alemaigne" vendait-il 700 aisselles «au bort de nef en l'eaue à l'Escluse", dont 400 de "grand bois" au prix de 41 s. gros le cent, destinées pour 200 d'entre elles à la résidence ducale de la Walle à Gand - que Jehan Ghisele de Gand livrait sur ses trois navires depuis la ville de Dam au rivage derrière la Walle pour $7 £$ parisis - et pour les 200 autres pour la galerie de l'hôtel de la Monnaie de Bruges ${ }^{7}$. Dans le compte des travaux effectués à l'hôtel ducal de Bruges de 1448 à 1452 apparaissent aussi les noms de marchands "alemands" résidant à Bruges, Jacques Danckaert, Simon Lanchals, Henry Tervacx, Simon Bruiter pour la vente «daissielles danemarche» au prix moyen de 50 s. le cent ${ }^{8}$.

La mise en ouvre et l'utilisation des lambris dans le décor de la demeure médiévale restent néanmoins mal connues ou tout au moins ont fait l'objet de peu de publications ${ }^{9}$. Le lambrissage («lambrochier», «lambreissier») par des "lambrous», "lambroys», "chambrillements», «chambrys»... des murs ("parois») comme des voûtes des pièces et salles des demeures aristocratiques fut pourtant un fait courant à partir de la fin du XIV ${ }^{\mathrm{e}}$ siècle au moins. Ce lambrissage concernait encore les édifices religieux et entre autres les voûtes en carène des églises lambrissées, nombreuses en Hainaut, Picardie, Bretagne, etc.

4. Pour un achat de l'abbaye de Saint-Vaast d'Arras en 1448-1449; H. Loriquet, J. Chavanon, Inventaire sommaire des archives départementales antérieures à 1790, Pasde-Calais, t. I, p. 108.

5. Archives départementales du Nord, B $9754,35 \mathrm{v}^{\circ}$ : «Pour 25 denemarches de bort de nef a faire les huiseries accatée a Gand ou mois de may l'an 75 pour les huiseriez de le sale au fuer de 6 s. le piece sont $7 £, 10$ s.».

6. J.-P. Sosson, Les travaux publics de la ville de Bruges (XIVe-XVe siècles), p. 102.

7. V. Leman, Les résidences des ducs de Bourgogne (1363-1477), p. 216.

8. J.-P. Sosson, Les travaux publics de la ville de Bruges (XIVe-XVe siècles), p. 111, n. 102.

9. A. Prévet («Structures et aménagements en bois dans l'architecture castrale médiévale à travers les collections du Centre de recherches sur les monuments historiques", p. 52 sq., n. 31) mentionne quelques lambris conservés. J.-J. Roman (Lambris $d u X V^{e}$ au milieu $d u X V I I^{e}$ siècle) en donne de nombreux relevés. 
Si la charpente de l'ancien palais synodal d'Auxerre est bien datée de 1248 , les lambris - que les auteurs supposent contemporains - n'ont pu l'être, car ils se situent en dehors d'un système référentiel de datation qui puisse leur correspondre ${ }^{10}$. Nous sommes donc confrontés à deux cas de figure, soit la charpente et lambrissage sont contemporains, soit le lambrissage est postérieur, mais de combien d'années? A contrario, on est parfois surpris de constater que des charpentes profilées en carène n'ont finalement pas été lambrissées.

\section{Apparition et diffusion du lambrissage}

Les mentions fréquentes dans les églises des $\mathrm{XI}^{\mathrm{e}}$ et $\mathrm{XII}^{\mathrm{e}}$ siècles de laquearia (terme traduit par lambris) recouvrant les plafonds et souvent décrits comme peints ${ }^{11}$ attestent une tradition antérieure de décor sur laquelle néanmoins nous sommes mal renseignés. Une rare mention en 1248, concernant la chambre de la reine au château de Villeneuve-le-Roi, nous assure de l'existence à cette date de lambris muraux ${ }^{12}$, lambris dont nous ne savons néanmoins pas s'ils avaient été réalisés avec des «bois de Danemarche».

À propos du palais de la Cité à Paris, construit sous Philippe le Bel (1285-1314), l'historien Sauval nous précise que les parois de la grande salle haute étaient systématiquement lambrissées de «bois de Danemarche»: ses deux vaisseaux, comme le montre la gravure de Jacques Androuet du Cerceau (1576), étaient de fait couverts d'une voûte aux berceaux lambrissés (fig. 2) que Sauval nous dit encore être peints et dorés ${ }^{13}$. Ce type de charpente lambrissée avec des voliges (ou aisselles)

Io. Ch. Locatelli, C. Lavier, "Étude de cas». Voir aussi dans le même ouvrage, P. Hoffsummer, «La forme du toit».

II. J. von Schlosser, Schriftquellen zur Geschichte der karolingischen Kunst, p. 5, 10 sq., 32 sq., 53, 133, 183, etc. V. Mortet, P. Deschamps, Recueil de textes relatifs à l'histoire de l'architecture et à la condition des architectes en France au Moyen Âge, XI ${ }^{e}-X I I^{e}$ siècles, p. 68, n. 4, p. 93 et passim.

I2. J. D. Guigniaut, N. de Wailly, Recueil des historiens des Gaules et de la France, p. 274: Pro domibus regis de Villa Nova affetatis, et camera reginae lambruisata, $32 £$; repris par V. Mortet, P. Deschamps, Recueil de textes relatifs à l'histoire de l'architecture et à la condition des architectes en France au Moyen Âge, XIe-XII e siècles, p. 256.

I3. H. Sauval, Histoire et recherches des antiquités de la ville de Paris, p. 3. 


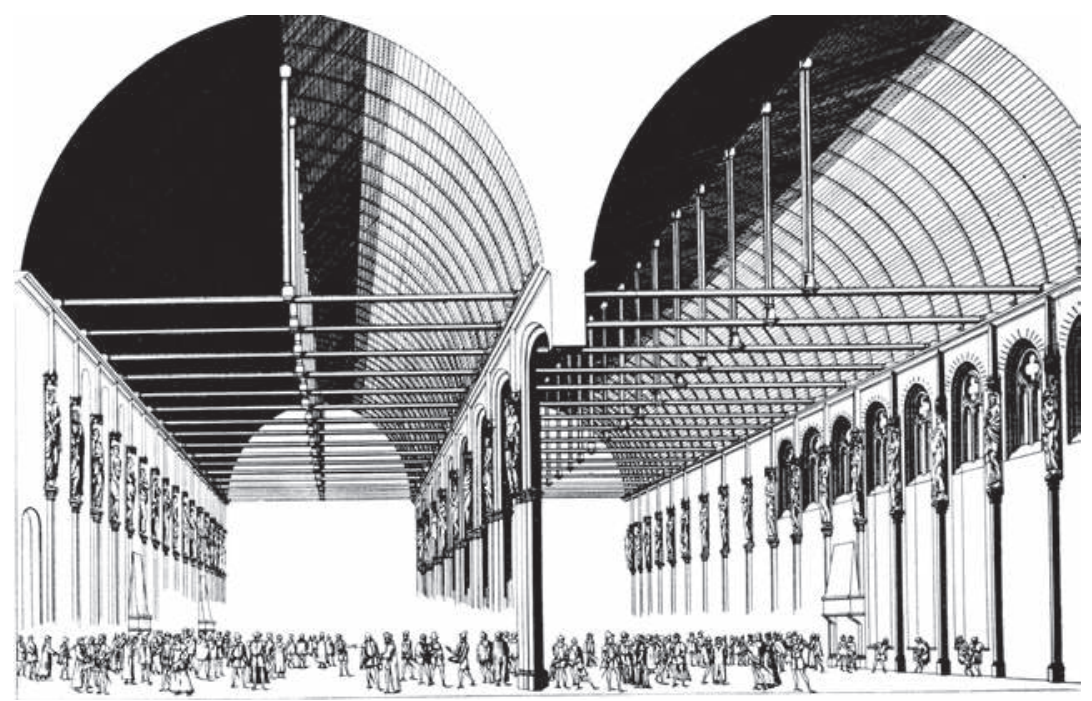

Fig. 2 - Paris, le Palais de la Cité, la grande salle, gravure de Jacques Androuet du Cerceau, 1576. Collection personnelle.

clouées sur des aisseliers courbes et dont les liaisons étaient cachées par des couvre-joints se retrouvait, entre autres, dans les grandes salles des châteaux de Montargis, Coucy-le-Château, Loches, Mehun-sur-Yèvre, Bourges, etc. à la fin du XIV ${ }^{\mathrm{e}}$ siècle. On serait néanmoins peut-être amené à douter du témoignage de l'historien Sauval, qui écrivait au XVII ${ }^{\mathrm{e}}$ siècle, sur la provenance des bois de la grande salle, si une autre source ne venait corroborer son témoignage.

De fait, Gervais du Bus, dans le Roman de Fauvel, évoque vers 1310 les murs lambrissés du palais de la Cité ${ }^{14}$ :

Le palaiz, ains fu lambroissiez

Trop cointement de fust d'Islande

A une taille espesse et grande

D'un bois qui n'est fendu n'ouvert,

Mès moult en soy clos et couvert.

Tremble y ot qui resembloit aune,

Mès tout fu vernicé de jaune,

Estincelant si clerement

Que nuz hom ne puet vraiement

I4. A. Langfors (texte établi par), Roman de Fauvel, v. 1370-1382. 
Veoir leens le ciel celestre,

S'il n'en yst par quelque fenestre,

Car les couleurs sont si ardans

Qu'il aveuglent les regardans

Le témoignage de Gervais du Bus est d'autant plus précieux qu'il fut, de 1312 à 1315, clerc et chapelain d'Enguerrand de Marigny (1260-1315), le principal ministre de Philippe le Bel, et par ailleurs maitre d'ouvrage (vers 1296-1310) du palais de la Cité. Sauval, comme Gervais du Bus, attestent donc que les voûtes lambrissées en bois d'Illande de la grande salle étaient en totalité ou en partie peintes: il en était de même au Logis royal de Loches (vers 1380) ${ }^{15}$ où la salle et la chambre étaient à l'origine couvertes par une charpente à berceau lambrissé ${ }^{16}$ dont les voliges étaient peintes. Le chantier du palais de la Cité a pu jouer un rôle particulier dans la diffusion de ce matériau en raison du prestige qui s'attachait à une réalisation royale et de la beauté de son décor, complété par les sculptures des rois de France accrochées aux colonnes ${ }^{17}$. Parmi les résidences des comtes d'Artois, celle de Conflans ${ }^{18}$ possédait dès 1320 plusieurs pièces lambrissées avec ce bois (salle, chambres, garde-robe) ${ }^{19}$.

Le logis royal du même palais de la Cité abritait encore la chambre «de bois d'Islande» dont l'aménagement devait dater du règne de Charles $\mathrm{V}$ (1364-1380). À l'Hôtel Saint-Pol, à Paris, réaménagé et reconstruit en partie par ce roi, les bains et étuves étaient "pavés de pierres de liais, fermés d'une porte de fer treillissé et entourés de lambris de bois d'Irlande; les cuves étoient de même bois d'Irlande, ornées tout autour de bossettes dorées et liées de cerceaux attachés avec des clous de cuivre dorés » ${ }^{20}$. Au château du Louvre, le quatrième niveau de la tour de la Librairie ou de la Fauconnerie vit en 1367 ses murs lambrissés de bois précieux («bois d'Illande»), par deux huchiers, Jacques du Parvis et Jean Grosbois ${ }^{21}$.

15. Indre-et-Loire.

I6. Aujourd'hui dissimulé par un plafond du $\mathrm{XV}^{\mathrm{e}}$ siècle.

17. E. Inglis, "Gothic architecture and a scholastic".

I8. Ancienne paroisse, actuellement rattachée à la commune de Charenton-le-Pont.

I9. O. Chapelot, B. Rieth, "Dénomination et répartition des espaces», p. 105.

20. H. Sauval, Histoire et recherches des antiquités de la ville de Paris, p. 280.

2I. A. Salamagne, "Le Louvre de Charles V». A. Le Roux de Lincy, "Comptes des dépenses faites par Charles V dans le château du Louvre des années 1364 à 1368 ", p. 764 sq. 
Un compte de 1364, aujourd'hui disparu, de Pierre Culdoe nous précise l'origine des bois:

A Robert Gringoire, pour avoir pris en un batel près la première porte du Louvre 480 pieces de bois d'Illande, et les porter et entasser dedans ledit chastel en une chambre, lesquels bois ont esté donnez au roi par le seneschal de Hainaut, pour les œuvres de son dit chastel, par marché fait 20 s. p. ${ }^{22}$.

Le sénéchal de Hainaut, Jean I ${ }^{\text {er }}$ de Werchin (mort en 1375), membre du conseil comtal de Hainaut, agissant probablement au nom du duc Albert de Bavière, comte de Hainaut et de Hollande, joua donc un rôle d'intermédiaire pour cette livraison faite par voie d'eau. Le transport devait avoir été réalisé directement depuis la Hollande jusqu'à Paris par le port de Rouen et avoir remonté la Seine pour être débarqué au Louvre même.

\section{Les anciens Pays-Bas}

Les comptabilités des anciens Pays-Bas montrent une mise en œuvre fréquente de ce bois dès la première moitié du XIVe siècle. Ainsi, au château de Mons en 1333-1334 pour des travaux à la maison dite d'Henri Loize $^{23}$, Jehan de Valenchiennes fournissait entre autres 50 «assielles de danemarche», pour 54 s., $1 \mathrm{~d}$. et Colart de le Loge 18 "assieles de Danemarche» et 75 lattes pour 27 s. Dans le même temps, l'usage de ce bois est attesté pour des pièces de mobilier d'église, crucifix (collégiale Saint-Amé de Douai, 1349-1350), des tableaux (ville de Lille, 1349-1350) ${ }^{24}$, etc. Un chirographe de 1374 d'un marché de charpenterie à exécuter dans une maison de la Couppe d'Or, au marché au blé ou grande-place de Douai, prescrivait entre autres travaux la reconstruction $\mathrm{du}$ «fausrans» (chevrons de tête de la ferme débordante d'un pignon) avec des planches de ce bois ${ }^{25}$. C'est sur l'ordre direct de la duchesse

22. Ibid., p. 678 et art. 106; original à la Bibliothèque de l'Arsenal, Paris, ms 6362 (101 ter) fo $54 \mathrm{r}^{\circ}$.

23. Archives départementales du Nord, B 7861, 1333-1334, $66 \mathrm{r}^{\circ}$.

24. Ch. Dehaisnes, Documents et extraits divers concernant l'histoire de l'art dans la Flandre, l'Artois et le Hainaut avant le XVe siècle, p. 388 sq.

25. G. Espinas, La vie urbaine à Douai au Moyen Âge, p. 467, nº1294: «le cliintre devens d'aissielles de Danemarche refendues». 
de Hainaut ${ }^{26}$ que le charpentier Pierre Katherine se rendait la première semaine de mai 1381 du Quesnoy à Valenciennes à la recherche de bois de Danemarche afin de lambrisser sa chambre vers les jardins. À défaut d'en trouver, il se rendit, mais après être revenu au Quesnoy pour obtenir l'accord de la duchesse et du Conseil ducal, à Tournai où fut trouvé le bois nécessaire: convoyé depuis cette dernière ville par l'Escaut jusqu'à Valenciennes, il fut ensuite acheminé par voie terrestre jusqu'au Quesnoy ${ }^{27}$. Au XVe siècle les mentions en sont très fréquentes dans les comptabilités de Flandre (dès 1398-1399 et 1404-1405 pour l'hôtel de la Poterne et la Salle de Lille ${ }^{28}$ ), Hainaut et Artois.

\section{La Bourgogne}

En Bourgogne, des mentions nombreuses en sont faites à partir de la fin du XIVe siècle, tout au moins sur les grands chantiers, par exemple en 1391 pour la chapelle et l'oratoire ducal de la chartreuse de Champmol fondée par Philippe le Hardi (1342-1404) ${ }^{29}$ :

A Pierre de Montmolin, pour la vendue et délivrance de 600 aiz de bois d'Illande de 9 piez de long et d'un espan ${ }^{30}$ de large la pièce, par luy prinses et achetées à Paris et admenez audit Champmol, pour mettre et convertir en lambroiz et endouveliz de la haulte chapelle et oratoire de mondit seigneur audit Champmol, au costé devers la montagne, et en plusieurs autres lieux nécessaires audit Champmol. Pour ce, paié a lui par sa quittance en la fin de laquelle la certifficacion des

26. Marguerite de Brieg $(1342 \dagger 1386)$, fille de Louis de Silésie-Liegnitz, duc de Brieg, et d'Agnès de Goglau.

27. Archives départementales du Nord, B 9027, 1381, fo $30 \mathrm{v}^{\circ}$.

28. V. Leman, Les résidences des ducs de Bourgogne (1363-1477), t. 2, p. 297, 302, $618 s q$.

29. Les lambris furent détruits à partir du XVII e siècle. C. Monget, La Chartreuse de Dijon d'après les documents des Archives de Dijon, p. 59 et 223; H. David, Claus Sluter, p. 46 sq. Pour la chapelle, voir R. Prochno, Die Kartause von Champmol, p. 154-167 et p. 157 pour les travaux de lambrissage.

30. Empan: $24-25 \mathrm{~cm}$. 
diz maistre Belin et Regnaudot donnée le $24^{\mathrm{e}}$ jour d'aoust 1391 est contenue... 60 frans $^{31}$.

La voûte de la chapelle, lambrissée de 13'500 planches, fut décorée par le peintre Jean de Beaumetz au début de l'année 1388, de "lymandes» ou bandes peintes dessinant des doubleaux et des ogives au chevet; des écus aux armes ducales peints sur étoffe et collés sur les bandes s'arrêtaient sur une litre peinte au sommet du mur portant les armes ducales ${ }^{32}$. Mais curieusement, dès les années 1375, les bois dits "d'Illande» pour les lambris de la chambre du futur Jean sans Peur au palais de Dijon ne provenaient pas de Livonie, mais de la forêt d'Argilly, au sud de Dijon ${ }^{33}$. Même provenance au château bourguignon de Germolles, pour ceux posés entre 1385 et 1405 sur la charpente du logis datée de 1385 (fig. 3) ${ }^{34}$. C’est donc que les spécificités propres au débitage du bois d'Illande avaient été adoptées et avaient laissé leur nom aux lambris ordinaires.

\section{Les chantiers de Jean de Berry (1369-1416)}

L'œuvre constructive réalisée entre 1380 et 1410 par Jean de Berry (13401416), frère du roi de France Charles V, est considérable. On peut penser que le duc adopta d'abord ce type de décor dans ses demeures parisiennes, dont l'hôtel de Nesle reçu en 1380 de Charles VI et qu'il agrandit «de jardins, de galleries, d'appartemens superbes et commodes» 35 . Au palais de Bourges (vers 1380-1386), capitale de son apanage en Berry, le bois d'Illande fut probablement employé pour les berceaux lambrissés couvrant la grande salle; au palais de Poitiers, le lambrissage du petit galetas et de la grande salle en bois fut exécuté par le huchier

31. C. Monget, La Chartreuse de Dijon d'après les documents des Archives de Dijon, p. 223.

32. M. Richard-Rivoire, "Le décor sur bois à la chartreuse de Champmol au temps des ducs de Valois", p. 249 sq.

33. Archives départementales de la Côte d'Or, B 4421, fo 23 ro, B 4423, fo 28 ro : source aimablement communiquée par Hervé Mouillebouche.

34. P. Beck (coordonné par), Vie de cour en Bourgogne à la fin du Moyen Âge, p. 32 et 86-98.

35. H. Sauval, Histoire et recherches des antiquités de la ville de Paris, p. 71 sq. 
Fig. 3 - Château de Germolles; taille et assemblage des lambris (cliché et dessin C. Locatelli et D. Pousset dans P. Beck [coordonné par], Vie de cour en Bourgogne à la fin du Moyen Âge, p. 93). 
parisien Guillaume Cirace, qui assumait lui-même la livraison en 1385 de 2000 planches de bois d'Illande ${ }^{36}$. À partir de 1383, les salles (grande salle, salle de parement et salle de retrait du corps de logis neuf), mais encore la voûte de la sacristie de la chapelle et les galeries supérieures du château du Clain dans la même ville, furent lambrissées de bois d'Illande livré par un charpentier de Lusignan ou venant du palais ${ }^{37}$. Au château de Lusignan ${ }^{38}$, si les travaux initiaux furent réalisés d'abord avec un matériau local - celui de la forêt de Coulombiers -, il fut rapidement abandonné au profit du bois d'Illande que le huchier parisien Guillaume Cirace faisait acheminer par voie maritime ${ }^{39}$.

Au château de Mehun-sur-Yèvre ${ }^{40}$, le lambrissage concerna la voûte en berceau de la grande salle et le troisième niveau de la tour maîtresse, réalisé néanmoins postérieurement à son aménagement vers $1390^{41}$. Les traces visibles sur la tour nord-ouest de réserves dans les maçonneries prouvent encore que les pièces des parties hautes étaient aussi couvertes de voûtes en bois lambrissées (fig. 4).

Au château de Poitiers, deux transports de bois d'Illande depuis le port de Niort (à $75 \mathrm{~km}$ par voie terrestre) sont mentionnés les 19 mars et 16 juillet $1386^{42}$ : le port de Niort constituait le départ de la navigation sur la Sèvre Niortaise jusqu'au port de Charron ou Marans. Ces bois qui provenaient probablement du port de Bruges étaient convoyés jusqu'à La Rochelle avant de parcourir par voie d'eau ou de terre $110 \mathrm{~km}$ jusqu'à Lusignan, $140 \mathrm{~km}$ jusqu'à Poitiers, outre les 600 miles de Bruges à La Rochelle. Le huchier parisien Guillaume Cirace ou Sirasse, qui succéda à Jean Brunet dès octobre 1384 sur le chantier de Poitiers, restera au service du duc de Berry jusqu'au début des années $1390^{43}$. Il était probablement apparenté aux huchiers du même nom qui travaillèrent pour les œuvres royales dès le règne de Jean II le Bon, ainsi de Jacques Sirasse qui recevait en 1361 la somme de 60 francs or "pour faire l'oratoire de nostre chapelle d'en haut de nostre hostel lez St. Paul» - alors l'hôtel

36. Th. Rapin, Les chantiers de Jean de France, duc de Berry, p. 39 et 65.

37. Ibid., p. 192 sq., 198, 205 et 225.

38. Département de la Vienne.

39. Ibid., p. 377 sq.

40. Département du Cher.

4I. Ph. Bon, «Un écrin de dentelle et de couleur», p. 244 sq.

42. Th. Rapin, Les chantiers de Jean de France, duc de Berry, p. 205, n. 1187.

43. Ibid., p. 377. 


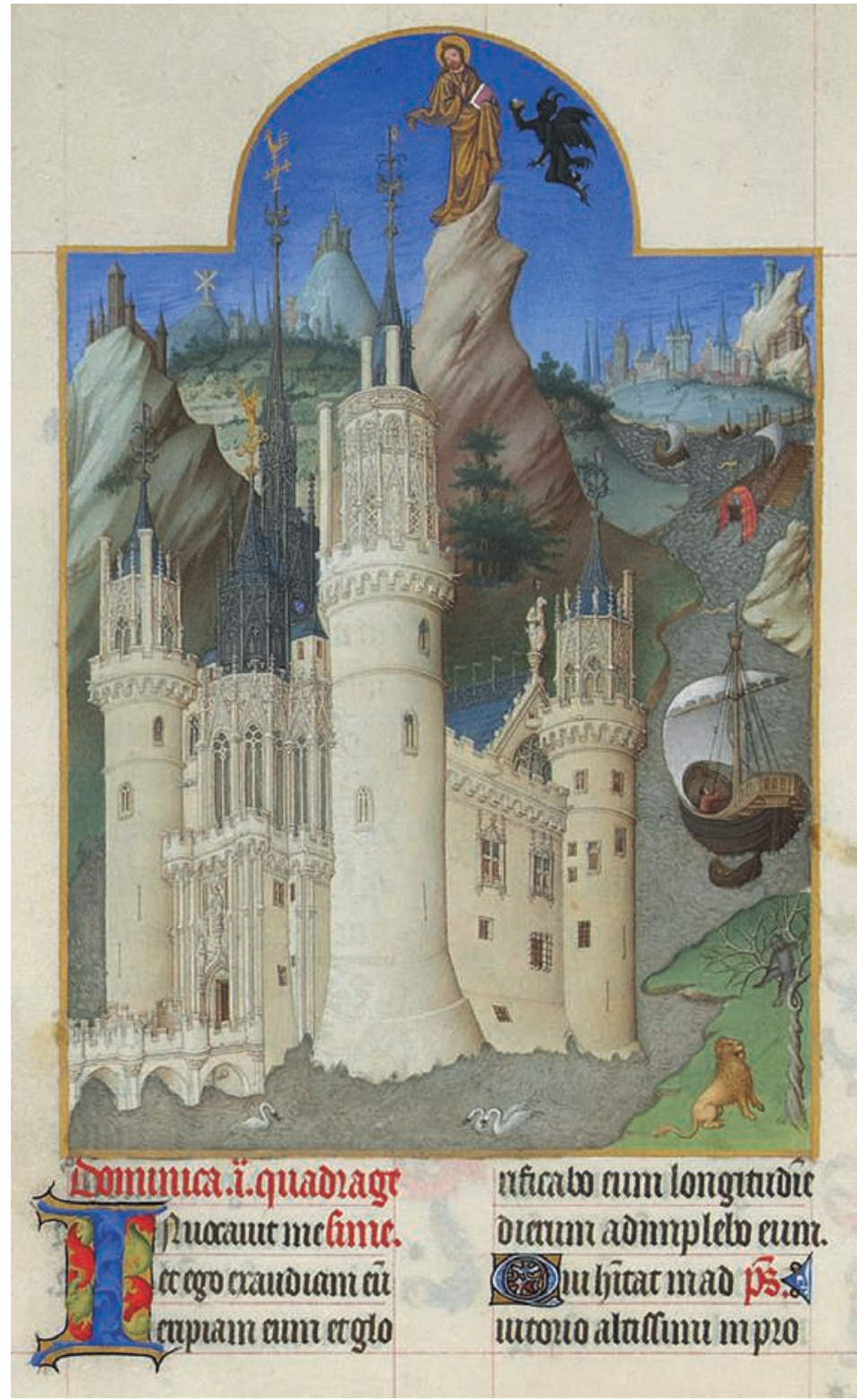

Fig. 4 - Mehun-sur-Yèvre: ainsi que la tour du nord-ouest, les deux autres tours à double couronnement devaient avoir leur dernier niveau lambrissé. Les Très Riches Heures du duc de Berry, La Tentation du Christ. Vers 1411-1416. Musée Condé Chantilly, Ms. 65, f. 161v. Photographie R.M.N. / R.-G. Ojéda, domaine public, Wikimedia Commons. 


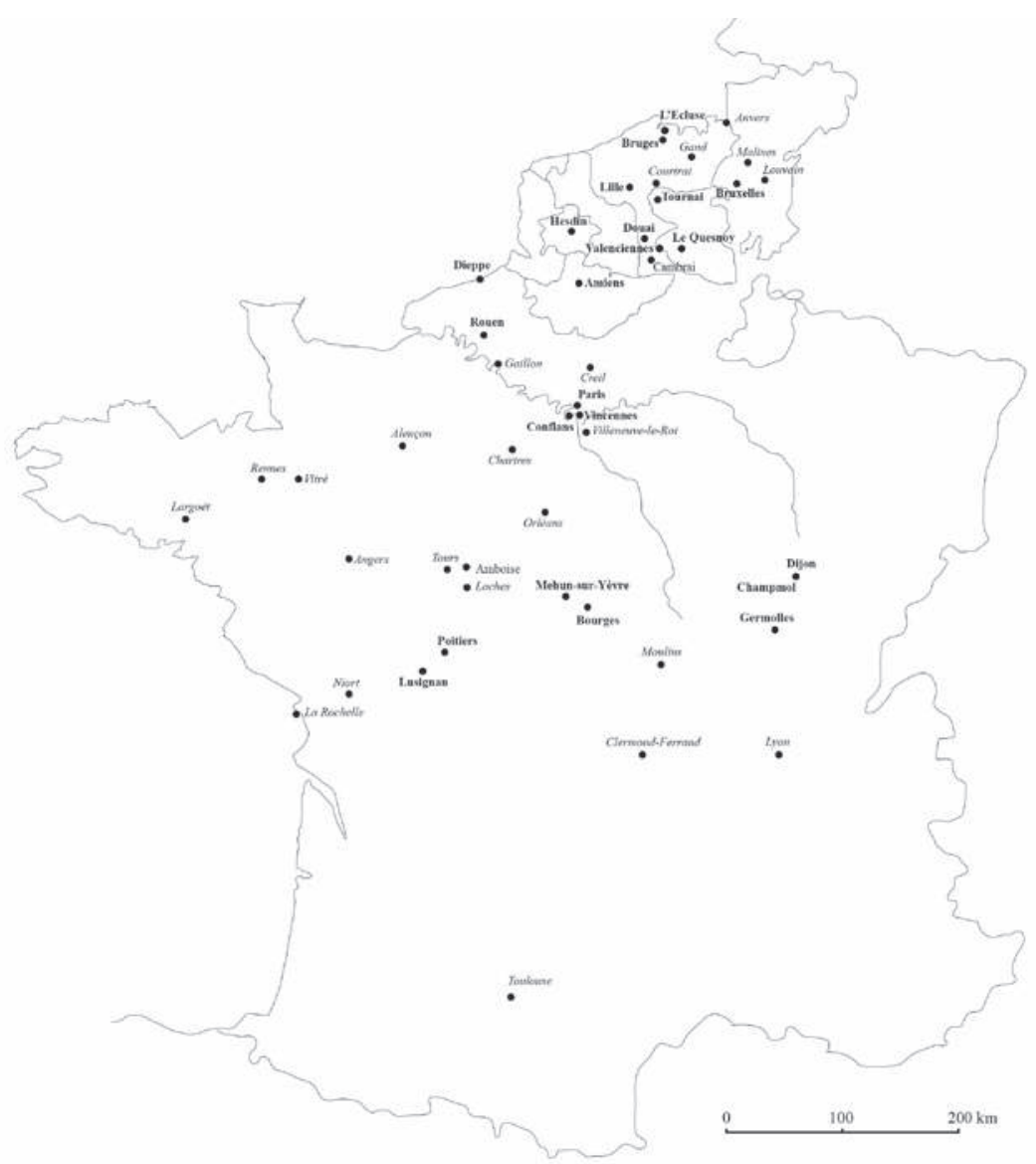

Fig. 5 - Carte des noms de lieux cités (en gras mentions concernant le bois d'Illande ou de Danemarche; en italique villes ou sites repères). (c) A. Salamagne.

d'Étampes ${ }^{44}$-, à Marie Cirasse qui réalisait du mobilier pour la salle de la reine au Louvre vers 1365 ou à Philippe Sirasse qui confectionnait «de bois d'Illande un estuy pour hebergier l'orloge M. le Dalphin qui sonne les oeres" pour le même lieu ${ }^{45}$. En 1406, Guillaume Sirasse qualifié de «bourgeois et huchier à Paris» obtenait un important marché

44. F. Bournon, «L'hôtel royal de Saint-Pol», p. 77.

45. A. Le Roux de Lincy, "Comptes des dépenses faites par Charles V dans le château du Louvre des années 1364 à 1368», p. 769, art. 124. 
de mobilier (d'un montant de 200 écus) pour le Parlement de Paris (les bancs et les parquets de la grande chambre) ${ }^{46}$. Après la répression cabochienne et la reprise en main du pouvoir du parti armagnac en 1413, Guillaume Cirasse fut nommé par le duc de Berry prévôt des marchands de la ville de Paris: il est désigné à cette occasion comme «charpentier du cimetière $S$. Jean " et "quartenier", c'est-à-dire capitaine de la milice urbaine d'un quartier de Paris ${ }^{47}$. Il résidait donc près du cimetière de Saint-Jean-en-Grève, voisin de la place de Grève et du centre du pouvoir communal parisien. Guillaume Cirasse avait son atelier situé à proximité des chantiers sur lesquels il intervint, le palais de la Cité et les hôtels du duc de Berry. Fin 1413 ou début 1414, il recevait encore commande de ce dernier pour son hôtel de Giac de différentes pièces de mobilier, dont certaines marquetées de bois d'Illande ${ }^{48}$.

\section{La diffusion en Normandie}

En Normandie, l'utilisation du bois de Danemarche semble avoir été plus ponctuelle; si son usage est attesté au château de Tancarville en 1410-1411 ${ }^{49}$, il faut attendre ensuite les années 1466 pour une seconde mention, celle des stalles et de la chaire épiscopale de la cathédrale de Rouen, en "bors d'Illande», œuvre de deux Hollandais, Guérard le Jeune et Frediq Franzone ${ }^{50}$. La diffusion du bois a pu être favorisée dès 1450 par l'arrivée en Normandie de sculpteurs de bois d'origine flamande à Rouen, ainsi de Laurent Hisbre ou encore de Paul Mosselman ${ }^{51}$. Les bois déchargés sur les quais de la Seine au port de Rouen provenaient

46. E. Boutaric, Recherches archéologiques sur le Palais de Justice de Paris principalement sur la partie consacrée au parlement depuis l'origine jusqu'à la mort de Charles VI (1422), p. $47 s q$.

47. J.-F. Michaud, J.-J.-F. Poujoulat, Histoire de Charles VI, roy de France, par Jean Juvénal des Ursins, p. 487 sq.

48. Détail dans A. de Champeaux, P. Gauchery, Les travaux d'art exécutés pour Jean de France duc de Berry, p. 30 et 188.

49. Seine-Maritime. Ph. Lardin, "L'utilisation du bois au château de Tancarville (Seine-Maritime) au cours du XVe siècle», p. 141 ; J. Mesqui, Le château de Tancarville, p. 39, 91 et 95 .

50. Ph. Lardin, "Le chantier des stalles de la cathédrale de Rouen (1457-1471)", p. 50.

5I. Ibid., p. 44. 
donc directement de Bruges par voie maritime (250 miles). Au $\mathrm{XV}^{\mathrm{e}}$ siècle, Dieppe recevait aussi des «bois de Flandre», de Prusse et de Scandinavie, transitant dans le complexe portuaire brugeois ${ }^{52}$. Si ailleurs, en Val de Loire comme à Orléans, il est fait mention du lambrissage par Pierre Piehen, dit Labieunoys, de la chambre aux orties et de la grande salle du logis ducal du Châtelet d'Orléans en remplacement de "celui qui y estoit pourry et ne valoit riens", l'origine du bois n'est pas indiquée ${ }^{53}$. Il en est de même pour d'autres régions, en Bretagne, en Alsace ${ }^{54}$... Par contre, le bois de Danemarche ne semble pas avoir été mis en ouvre sur les chantiers d'Auvergne ${ }^{55}$ trop éloignés assurément des grands centres de redistribution.

\section{Conclusion}

La connaissance que nous pouvons avoir du lambrissage des pièces des demeures aristocratiques aux $\mathrm{XIV}^{\mathrm{e}}$ et $\mathrm{XV}^{\mathrm{e}}$ siècles résulte d'abord de l'analyse des comptabilités, même si des exemples en sont conservés par endroit (ainsi aux châteaux de Chateaudun, Vitré, etc.) ou si les traces en sont apparentes par les saignées laissées dans les murs (Mehun-surYèvre, palais Rihour à Lille, etc.). Du XIVe au XVI siècle les mentions de bois de Danemarche sont très nombreuses dans les comptabilités des anciens Pays-Bas qui attestent à la fois que ces régions en constituèrent le grand centre redistributeur, mais aussi en imposèrent la mode. Cette dernière s'implanta dans l'architecture royale française au moins dès 1300 , relayée au XIV ${ }^{\mathrm{e}}$ siècle par les contacts étroits entretenus en particulier par la dynastie des comtes d'Artois ou de Hainaut-Hollande avec la cour de France, pour se diffuser ensuite dans les châteaux et demeures de Charles V et de ses frères.

52. M. Mollat, «Les constructions navales à Dieppe», p. 134.

53. É. Roux-Capron et al., ORLÉANS, no 80 quai du Châtelet, p. 125.

54. Pour les lambris d'une Stube datés de 1377 au 4 rue des Tonneliers à Dambachla-Ville; cf. M. Werlé, M. Seiller, Ch. Dormoy, «L'apport de l'archéologie du bâti à la connaissance des habitats médiévaux urbains en Alsace», p. 129. Ils deviennent plus courants au $\mathrm{XV}^{\mathrm{e}}$ siècle.

55. Tout au moins, B. Phalip (Charpentiers et couvreurs) n'en fait pas mention. 
Les chambres, les études, les galeries, les chapelles privées, voire les étuves, furent les pièces les plus couramment concernées par le couvrement en bois précieux, partiel ou total, des murs.

Pour le domaine germanique si le lambrissage des pièces des demeures aristocratiques était courant, le qualificatif équivalent à "bois de Danemarche" n'est pas répertorié et laisse penser qu'aucune distinction n'était faite avec un bois de chêne ordinaire. En Angleterre par contre, l'équivalent pourrait être désigné sous le terme de «bord de Alemain» (1275) mais la dénomination la plus courante est celle de "estreche board» pour qualifier les bois importés de la Baltique, dont ceux venant de Riga («estrichbord de Rygald»). On a encore relevé la mention pour l'année 1369 de «tables destland» (proche donc du français «islande») livrées pour la confection de portes et de volets ${ }^{56}$.

Reste à se poser la question du pourquoi de l'importation de ces bois de la Baltique et de leurs usages particuliers, lambris non seulement, mais aussi mobilier précieux, stalles voire portes et fenêtres des demeures aristocratiques? On soulignera d'abord la qualité supérieure de ces bois, leur capacité à ne pas se voiler qui procédait de leur débitage particulier qui consistant à les refendre sur maille, c'est-à-dire dans le sens des rayons médullaires. C'est peut-être la raison pour laquelle, on l'a vu pour la Bourgogne, le qualificatif de "danemarche" fut appliqué à des bois locaux, mais qui avaient probablement été débités avec cette technique.

\section{Alain Salamagne \\ Université de Tours}

56. L. F. Salzman, Building in England down to 1540, p. 245 sq. 


\section{BIBLIOGRAPHIE}

BeCK, Patrice (coordonné par), Vie de cour en Bourgogne à la fin du Moyen Âge, Saint-Cyr-sur-Loire, Allan Sutton, 2002.

Bon, Philippe, "Un écrin de dentelle et de couleur. La notion d'ensemble décoratif: la résidence princière de Mehun-sur-Yèvre", in Cadres de vie et manières d'habiter (XII ${ }^{e}-X V I^{e}$ siècles). Actes du VIII congrès international de la Société d'Archéologie Médiévale, Paris 11-13 octobre 2001, éd. par Danièle Alexandre-Bidon, Françoise Piponnier, Jean-Michel Poisson, Caen, Publications du CRAHM, 2006, p. 239-247.

Bournon, Fernand, "L'hôtel royal de Saint-Pol", Mémoires de la société de l'histoire de Paris et de l'Île-de-France, t. VI, 1879, p. 54-179.

Boutaric, Edgard, Recherches archéologiques sur le Palais de Justice de Paris principalement sur la partie consacrée au parlement depuis l'origine jusqu'à la mort de Charles VI (1422), Paris, Charles Lahure, 1862.

Champeaux, Alfred de, Gauchery, Paul, Les travaux d'art exécutés pour Jean de France duc de Berry, Paris, Honoré Champion, 1894.

Chapelot, Jean, Pousset, Didier, «Les lambris du donjon», Vincennes, du manoir capétien à la résidence de Charles $V$, Dossiers d'Archéologie, 289 (2004), p. 84-89.

Chapelot, Odette, Rieth, Bénédicte, «Dénomination et répartition des espaces: exemples de deux résidences princières en Île-deFrance: le manoir de Conflans et l'hôtel d'Artois", in Cadre de vie et manières d'habiter (XII ${ }^{e}-X V I^{e}$ siècle). Actes du VIII e congrès international de la Société d'Archéologie Médiévale, Paris 11-13 octobre 2001, éd. par Danièle Alexandre-Bidon, Françoise Piponnier, Jean-Michel Poisson, Caen, Publications du CRAHM, 2006, p. 103-116.

David, Henri, Claus Sluter, Paris, Pierre Tisné, 1951.

Dehaisnes, Chrétien, Documents et extraits divers concernant l'histoire de l'art dans la Flandre, l'Artois et le Hainaut avant le XVe siècle. Première partie: 627-1373, Lille, Imprimerie L. Danel, 1886. 
Espinas, Georges, La vie urbaine à Douai au Moyen Âge, t. 4, Paris, Picard, 1913.

Guigniaut, Joseph Daniel, Wailly, Natalis de, Recueil des historiens des Gaules et de la France, t. XXI, Paris, Imprimerie Impériale, 1855. Hoffsummer, Patrick, "La forme du toit", in Charpentes du XI au $X I X^{e}$ siècle. Typologie et évolution en France du Nord et en Belgique, éd. par Patrick Hoffsummer, Jeannie Mayer, Paris, Monum, Éditions du patrimoine, 2002, p. 155-158 (Cahiers du Patrimoine, 62).

INGLIS, Erik, "Gothic architecture and a scholastic: Jean de Jandun's Tractatus de Laudibus Parisius (1323)», Gesta, XLII/1 (2003), p. 63-85.

Langfors, Arthur (texte établi par), Roman de Fauvel, Paris, FirminDidot, 1914.

Lardin, Philippe, Les chantiers du bâtiment en Normandie orientale à la fin du Moyen Âge. Les matériaux et les hommes, thèse de l'Université de Rouen, 1995.

—, "Le chantier des stalles de la cathédrale de Rouen (1457-1471)», in Les stalles de la cathédrale de Rouen. Histoire et iconographie, éd. par Elaine C. Block, Frédéric Billiet, Rouen, Publications de l'Université de Rouen, 2003, p. 33-71.

—, "L'utilisation du bois au château de Tancarville (Seine-Maritime) au cours du XV siècle", in Le bois dans le château de pierre au Moyen Âge. Actes du colloque de Lons-le-Saunier, 23-25 octobre 1997, éd. par Jean-Michel Poisson, Jean-Jacques Schwien, Besançon, Presses Universitaires Franc-comtoises, 2003, p. $129-150$.

Leman, Victorien, Les résidences des ducs de Bourgogne (1363-1477): Habitat et cadre de vie princiers à la fin du Moyen Âge, thèse de l'Université d'Amiens, 2017.

Le Roux de Lincy, Antoine, "Comptes des dépenses faites par Charles V dans le château du Louvre des années 1364 à 1368 ", Revue archéologique, 8 (1851-1852), p. 670-691 et 760-772.

Locatelli, Christian, Lavier, Catherine, "Étude de cas: les charpentes de l'ancien palais synodal et de la cathédrale Saint-Étienne d'Auxerre", in Charpentes duXI' au XIX' siècle. Typologie et évolution en France du Nord et en Belgique, éd. par Patrick Hoffsummer, 
Jeannie Mayer, Paris, Monum, Éditions du patrimoine, 2002, p. 142-145 (Cahiers du Patrimoine, 62).

Loriquet, Henri, Chavanon, Jules, Inventaire sommaire des archives départementales antérieures à 1790, Pas-de-Calais. Archives ecclésiastiques - série H, t. I, Art 1-851, Arras, Imprimerie moderne, 1902.

Mesqui, Jean, Le château de Tancarville. Histoire et architecture, Paris, Société française d'archéologie, supplément au Bulletin monumental, 2007.

Michaud, Joseph-François, Poujoulat, Jean-Joseph-François, Histoire de Charles VI, roy de France, par Jean Juvénal des Ursins, Paris, Éditeur du Commentaire analytique du Code civil, 1836 (Nouvelle collection de mémoires pour servir à l'histoire de France, 2).

Mollat, Michel, «Les constructions navales à Dieppe», Bulletin philologique et historique du C.T.H.S, 1966, (1968), p. 131-141.

Monget, Cyprien, La Chartreuse de Dijon d'après les documents des Archives de Dijon, Montreuil-sur-Mer, Imprimerie Notre-Damedes-Prés, t. 1, 1898.

Mortet, Victor, Deschamps, Paul, Recueil de textes relatifs à l'histoire de l'architecture et à la condition des architectes en France au Moyen Âge, XIe-XIII siècles, vol. 2, Paris, Picard, 1929.

Phalip, Bruno, Charpentiers et couvreurs: l'Auvergne médiévale et ses marges, Lyon, Alpara, 2017.

PrÉVET, Alain, «Structures et aménagements en bois dans l'architecture castrale médiévale à travers les collections du Centre de recherches sur les monuments historiques", in Le bois dans le château de pierre au Moyen Âge. Actes du colloque de Lons-le-Saunier, 23-25 octobre 1997, éd. par Jean-Michel Poisson, Jean-Jacques Schwien, Besançon, Presses Universitaires Franc-comtoises, 2003, p. 45-59.

Prochno, Renate, Die Kartause von Champmol. Grablege der burgundischen Herzöge 1364-1477, Berlin, Akademie Verlag, 2002.

Rapin, Thomas, Les chantiers de Jean de France, duc de Berry: maîtrise d'ouvrage et architecture à la fin du XIV siècle, thèse de l'Université de Poitiers, 2010.

Richard-Rivoire, Monique, "Le décor sur bois à la chartreuse de Champmol au temps des ducs de Valois", in Actes des Journées 
internationales Claus Sluter (septembre 1990), Dijon, Association Claus Sluter, 1992, p. 249-257.

Roman, Jean-Jacques, Lambris du XV $V^{e}$ au milieu du XVII siècle, Paris, Éditions du patrimoine, Centre des monuments nationaux, 2015. Roux-Capron, Émilie et al., ORLÉANS, $n^{\circ} 80$ quai du Châtelet. Site 45.234.246. Rapport d'opération préventive de fouille archéologique, Volume 1: résultats archéologiques, DRAC/SRA Centre, Ville d'Orléans, 2015.

Salamagne, Alain, "Le Louvre de Charles V", in Le palais et son décor au temps de Jean de Berry, éd. par Alain Salamagne, Tours, Presses Universitaires François Rabelais, 2010, p. 75-138.

Salzman, Louis Francis, Building in England down to 1540. A documentary history, Oxford, Clarendon Press, 1952.

Sauval, Henri, Histoire et recherches des antiquités de la ville de Paris, Paris, chez Charles Moette et Jacques Chardon, 1724, t. 2.

Schlosser, Julius von, Schriftquellen zur Geschichte der karolingischen Kunst, Vienne, Verlag von Carl Graeser, 1896.

Sosson, Jean-Pierre, Les travaux publics de la ville de Bruges (XIV ${ }^{e_{-}}$ $X V^{e}$ siècles), Bruxelles, Crédit Communal de Belgique, 1977.

Werlé, Maxime, Seiller, Maurice, Dormoy, Christian, «Lapport de l'archéologie du bâti à la connaissance des habitats médiévaux urbains en Alsace", Bilan scientifique de la région Alsace, hors série 2/2 (2006), p. 123-131. 